\title{
EDITORIAL
}

\section{Why our readers should concern themselves with their patients' lifestyle and diet}

\author{
Prostate Cancer and Prostatic Diseases (2006) 9, 103-104. \\ doi:10.1038/s.pcan.4500886
}

'Lifestyle' is currently a buzz word. Traditionally clinicians who focus on the prostate have steered clear of lifestyle-related issues, concerning themselves instead with lower urinary tract symptoms (LUTS), prostatespecific antigen (PSA) levels and associated erectile dysfunction (ED). However, in the light of recent uncertainties about the reliability of PSA as a cancer marker and anxieties over the risks of over-diagnosis of prostate cancer as well as the demographic trends towards an increasingly obese population, ${ }^{1}$ there is now an argument for all of us to start taking a broader view.

More and more men nowadays are overweight and the typical central obesity that they suffer is strongly linked with the metabolic syndrome and diabetes mellitus. Lipid levels in males are also often too high, carrying increased risks of cardiovascular disease and premature death. Obesity has also been proposed as a risk factor for prostate cancer in a paper published in the last issue of Prostate Cancer and Prostatic Diseases, ${ }^{2}$ and it also increases the risks of complications following surgical interventions for the disease.

As penile erection is a vasodilatory event, it is not surprising that men who suffer from ED not infrequently have more widespread endothelial disease. ${ }^{3}$ Recognition of concomitant hypertension, coronary artery or carotid vessel atheroma can trigger targeted interventions that may prevent future myocardial infarction (MI) or stroke.

There is, therefore, an argument for urologists and other readers of this journal, when faced with patients presenting with commonplace urological complaints, to consider measuring height, weight and abdominal girth as well as blood pressure, fasting lipids and blood sugar. In men with prostate problems and ED, carotid artery Doppler studies and possibly coronary artery computerised tomography (CT) to assess cardiac vessel calcification may be worth considering with referral to a cardiologist or vascular surgeon if significant lesions are detected.

An increased waist circumference and/or body mass index (BMI) is significantly associated with increased cardiovascular risk as well as all the other deleterious components of the metabolic syndrome including diabetes mellitus (Figure 1). Exhortation about a sensible diet and increased exercise may therefore be in order. If urologists feel uneasy with this new stance they might be encouraged by the recent finding of Giovannucci et al. ${ }^{4}$ that men who undertook more than $3 \mathrm{~h}$ of vigorous exercise per week reduced their risk of developing advanced prostate cancer or dying from the disease by around $70 \%$.

If total and low-density lipoprotein (LDL) cholesterol are significantly raised there is good evidence that

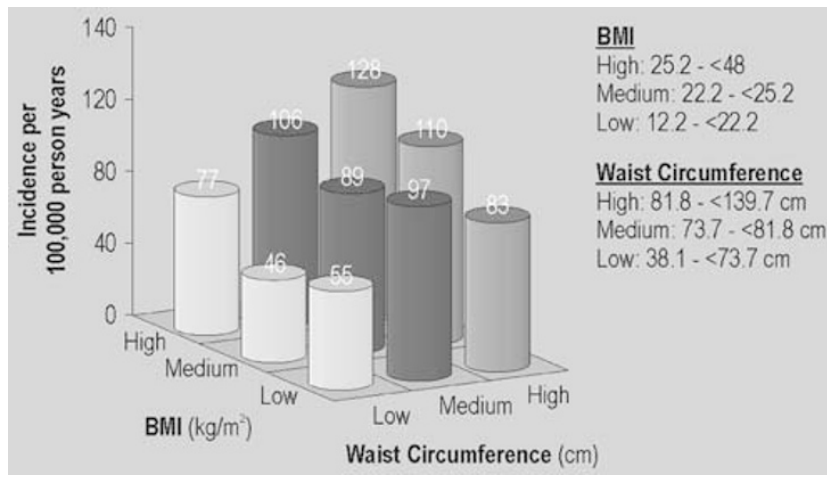

Figure 1 Incidence of coronary heart disease by waist circumference and BMI.

lowering these with a statin will result in a significant reduction in cardiovascular risk. A recent meta-analysis revealed that a $1 \mathrm{mmol} / 1$ reduction in LDL cholesterol translated into an $18 \%$ reduction of MI or stroke. Intriguingly, there is emerging evidence that statins may also reduce PSA levels ${ }^{G}$ and offer some chemopreventative protection against prostate cancer. ${ }^{7}$

Sometimes unwittingly, urologists have been prescribing agents that afford some cardioprotective effect. Several of the alpha blockers used so extensively in the management of LUTS resulting from benign prostatic hyperplasia $(\mathrm{BPH})$ result in reduction of blood pressure in hypertensive men as well as a reduction in LDL cholesterol. ${ }^{8}$ Interestingly they also appear to have a mildly beneficial effect on erectile function. ${ }^{9}$

Taking all these factors in to account the way ahead seems clear. Instead of focusing exclusively on prostatic complaints, perhaps we should consider taking a more holistic view and become the proponents of men's health. ${ }^{10}$ As an alternative to merely prescribing pharmacotherapy for LUTS or relentlessly pursuing the cause of a mildly elevated PSA, as well as referring our patients on to separate specialist hypertension, dyslipidaemia or diabetes clinics, we should consider taking a leaf out of the gynaecologists' book and address the overall issue of our patient's lifestyle and health. ${ }^{11}$ In a rapidly changing world this would not only offer prostate specialists a future that is less dependent on surgical or radiotherapuetic intervention, but one that provides our patients with an evidence-based pathway to achieve worthwhile improvement in both longevity and quality of life. This is the way we should like to take our new, revamped journal Prostate Cancer and Prostatic Diseases forward, we sincerely hope our readers will join our crusade.

R Kirby

The Prostate Centre, London, UK 


\section{References}

1 Vasan RS, Pencina MJ, Cobain M, Freiberg MS, D'Agostino RB. Estimated risks for developing obesity in the Framingham Heart Study. Ann Intern Med 2005; 143: 473-480.

2 Ribeiro R, Lopes C, Medeiros R. The link between obesity and prostate cancer: the leptin pathway and therapeutic perspectives. Prost Cancer Prost Diseases 2006; 9: 19-24.

3 Greenstein A, Chen J, Miller H, Matzkin H, Villa Y, Braf Z. Does severity of ischaemic coronary heart disease correlate with erectile dysfunction? Int J Impot Res 1997; 9 123-126.

4 Giovannucci EL, Liu Y, Leitzmann MJ, Stamfer MJ, Willett WC. A prospective study of physical activity and incident and fatal prostate cancer. Arch Int Med 2005; 165: 1005-1010.

5 Cholesterol Treatment Trialists Collaborators. Efficacy and safety of cholesterol-lowering treatment: prospective meta-analysis of data from 90056 participants in 14 randomized trials of statins. Lancet 2005; 366: 1267-1278.
6 Shannon J, Tewoderos S, Garzotto M, Beer TM, Derenek R, Palma A et al. Statins and prostate cancer risk: a case control study. Am J Epidemiol 2005; 162: 318-325.

7 Cyrus-David M, Weinberg A, Thompson T, Kadmon D. The effect of statins on serum prostate specific antigen levels in a cohort of airline pilots: a preliminary report. J Urol 2005; 173 : 1923-1925.

8 Kirby RS. Doxazosin in benign prostatic hyperplasia:effects on blood pressure in normotensive and hypertensive men. Urology 1995; 46: 182-186.

9 Kirby RS, O'Leary MP. Carson C Efficacy of extended-release doxazosin and doxazosin in patients with concomitant benign prostatic hyperplasia and sexual dysfunction. BJU Int 2005; 95: 103-109.

10 Kirby RS. The urologist as an advocate of men's health. BJU Int 2005; 95: 929.

11 Kirby RS, Kirby MG. The urologist as an advocate of men's health: 10 suggested steps toward helping patient's achieve better overall health. Urology 2005; 66 (suppl 5A): 52-56. 